\title{
Sharper angle, higher risk? The effect of cutting angle on knee mechanics in invasion sport athletes
}

\author{
Mervin J. Schreurs ${ }^{a}$, Anne Benjaminse ${ }^{\mathrm{a}, \mathrm{b}, *}$, Koen A.P.M. Lemmink ${ }^{\mathrm{a}}$ \\ ${ }^{a}$ Center for Human Movement Science, University Medical Center Groningen, University of Groningen, Antonius Deusinglaan 1, 9713 AV Groningen, The Netherlands \\ ${ }^{\mathrm{b}}$ School of Sport Studies, Hanze University Groningen, Zernikeplein 17, 9747 AS Groningen, The Netherlands
}

\section{A R T I C L E I N F O}

\section{Article history:}

Accepted 19 August 2017

Available online $\mathrm{xxxx}$

\section{Keywords:}

Cutting

Knee

Biomechanics

Anterior cruciate ligament

\begin{abstract}
A B S T R A C T
Introduction: Cutting is an important skill in team-sports, but unfortunately is also related to non-contact ACL injuries. The purpose was to examine knee kinetics and kinematics at different cutting angles. Material and methods: 13 males and 16 females performed cuts at different angles $\left(45^{\circ}, 90^{\circ}, 135^{\circ}\right.$ and $\left.180^{\circ}\right)$ at maximum speed. 3D kinematics and kinetics were collected. To determine differences across cutting angles $\left(45^{\circ}, 90^{\circ}, 135^{\circ}\right.$ and $\left.180^{\circ}\right)$ and sex (female, male), a $4 \times 2$ repeated measures ANOVA was conducted followed by post hoc comparisons (Bonferroni) with alpha level set at $\alpha \leq 0.05$ a priori.

Results: At all cutting angles, males showed greater knee flexion angles than females $(\mathrm{p}<0.01)$. Also, where males performed all cutting angles with no differences in the amount of knee flexion $-42.53^{\circ} \pm 8.95^{\circ}$, females decreased their knee flexion angle from $-40.6^{\circ} \pm 7.2^{\circ}$ when cutting at $45^{\circ}$ to $-36.81^{\circ} \pm 9.10^{\circ}$ when cutting at $90^{\circ}, 135^{\circ}$ and $180^{\circ}(\mathrm{p}<0.01)$. Knee flexion moment decreased for both sexes when cutting towards sharper angles $(\mathrm{p}<0.05)$. At $90^{\circ}, 135^{\circ}$ and $180^{\circ}$, males showed greater knee valgus moments than females. For both sexes, knee valgus moment increased towards the sharper cutting angles and then stabilized compared to the $45^{\circ}$ cutting angle $(p<0.01)$. Both females and males showed smaller vGRF when cutting to sharper angles $(\mathrm{p}<0.01)$.

Conclusion: It can be concluded that different cutting angles demand different knee kinematics and kinetics. Sharper cutting angles place the knee more at risk. However, females and males handle this differently, which has implications for injury prevention.
\end{abstract}

๑ 2017 Elsevier Ltd. All rights reserved.

\section{Introduction}

In many invasion sports (team games in which the purpose is to invade the opponent's territory while scoring points) success may rely on the ability of a player to make quick changes of direction (cutting). In addition agility (i.e. a rapid whole body movement with change of direction of speed or direction in response to a stimulus) is needed to block attacking movements or evade defenders (Sheppard and Young, 2006). Notational analysis showed that football players in the Football Association premier league frequently have to change direction with different cutting angles (Bloomfield et al., 2007a, b). Further earlier research has found that agility can discriminate between first and second division basketball players (Sekulic et al., 2016). Also, research showed that cutting is an important factor in talent identification (Reilly et al., 2000).

\footnotetext{
* Corresponding author at: Center for Human Movement Sciences, University of Groningen, Antonius Deusinglaan 1, 9713 AV Groningen, The Netherlands.

E-mail address: a.benjaminse@umcg.nl (A. Benjaminse).
}

However, these cutting manoeuvres are often associated with non-contact anterior cruciate ligament $(\mathrm{ACL})$ rupture (Cochrane et al., 2007; Olsen et al., 2004). In order to make a directional change an athlete first has to decelerate, then redirecting the body into the new direction, and finally accelerate into the new direction (Hase and Stein, 1999). During these deceleration-acceleration motions coupled with rapid change in direction, larger knee varus/valgus moments as well as lower knee flexion are identified as potential risk factors of non-contact ACL ruptures (McLean et al., 2004; Olsen et al., 2004; Leppanen et al., 2017).

As mentioned above, team-sport players have to change direction to different cutting angles. However, limited knowledge is available on the kinetic and kinematic during cutting at different angles. It is known that athletes use greater knee flexion angles when making a cut of $45^{\circ}$ compared to a $90^{\circ}$ cut (Havens and Sigward, 2015 a, b). Also, it has been found that knee flexion angle at peak stance does not differ between the angles of $45^{\circ}$ and $180^{\circ}$ (Cortes et al., 2011). Earlier research showed that athletes had greater knee valgus moments when cutting to a $110^{\circ}$ angle compared to a $45^{\circ}$ change of direction (Sigward et al., 2015). In 
addition, it was found that knee extension moment decreased in the $180^{\circ}$ task compared to the $45^{\circ}$ task, but varus/valgus moment increased (Cortes et al., 2011), which is unfavorable in terms of knee load. A pivot task, with $180^{\circ}$ of change in direction, might provide a more realistic representation of a soccer task (Greig, 2009). Considering the different motor control mechanisms necessary, the different demands placed on the body, it is imperative to examine multiple biomechanical risk factors depending on the task (Newell, 1996; Newell and Slifkin, 1998).

As far as the authors know these differences have never been examined in a whole range of different cutting angles within one study. Also the above mentioned studies used a predetermined speed. One might expect the knee kinematics and kinetics to change when athletes perform a cutting task at maximal effort.

Therefore, the aim of the current study was to investigate the effects of different cutting angles on knee kinematics and kinetics. It was hypothesized that cutting to sharper angles leads to increased knee joint loads.

\section{Methods}

\subsection{Participants}

Twenty-nine healthy team sport athletes $18-27$ years old participated in this study: 13 males $(22.5 \pm 2.3$ years, $190.2 \pm 7.7 \mathrm{~cm}$ and $80.1 \pm 10.3 \mathrm{~kg})$ and 16 females (21.9 \pm 2.5 years, $173.6 \pm 6.9 \mathrm{~cm}$ and $67.1 \pm 7.1 \mathrm{~kg}$ ). Based on previous research sample size was estimated for a minimal statistical power of $80 \%$ $(\alpha=0.05)$ (Havens and Sigward, $2015 \mathrm{a}, \mathrm{b})$. Given the variation of the dependent measures that were included in this study, 25 subjects were deemed adequate ( $\mathrm{G} *$ Power for Mac, version 3.1.2). G-power is a respected program to calculate the power of studies. We arrived at 25 subjects with an effect size (ES) of 0.25 for the medium effect of an ANOVA and an alpha of 0.05 . With 40 subject a power of 0.80 would be reached.

Participants were included if they: (1) participated in invasion team sports at least three times a week (two training sessions and one game), (2) older than eighteen years of age and (3) currently free of lower-limb injuries. Participants were excluded if they had an ACL injury or reconstruction in their lifetime. Prior to participation subjects received information about the goals, risks, and protocols of this study. All participants signed an informed consent before the test session. Procedures were approved by the University of Groningen Medical Ethics Committee (ID numbers: CCMO protocol number: NL24814.082.09, METc:2009.142).

\subsection{Subject preparation}

At the start of each test session anthropometric measures were taken prior to placement of 21 reflective markers of $14 \mathrm{~mm}$ in diameter placed according to the Vicon Plug-in-Gait + trunk marker set. After placing the markers, a static calibration trial was performed. Participants wore their own indoor shoes, thigh shorts and a tight shirt.

After the calibration trial the participants performed a standardized warming-up protocol. This warming-up consisted of jogging 3 laps around a standard volleyball court, 10 lunges, 5 jump-squats, sub maximal exercises with cutting movements and finally, several practice trials at full speed for all of the angles used in this study.

\subsection{Study protocol}

Testing took place from January 2016 to May 2016. Participants $(n=29)$ completed 50 trials in a laboratory setting on one day. One trial consisted of a 5 meter sprint towards a force plate, a cutting manoeuvre on the force plate, and a 5 meter sprint towards the endpoint (see Fig. 1). Five different conditions were examined in this study namely running straight ahead, and making cuts of $45^{\circ}, 90^{\circ}, 135^{\circ}$ and $180^{\circ}$. Each condition was completed 10 times. The trials were offered in blocks of 5 trials with the same angle. To prevent effects of testing order these blocks were randomized between participants. The participants got verbally instructed and encouraged to sprint at full speed from start till finish. During the practice trials the participants had ample time to decide where to exactly start running to assure correct foot placement on the force plate without targeting. This start was then marked with a tape on the floor, so that the participants did not have to think about it anymore during the following trials. We further instructed the participants to not place too much focus on the force plates. Also, if the participants missed the force plate in a certain trial, this trial was ignored in data analysis.

To prevent fatigue subjects got enough rest in between trials and blocks of trials. The rest was $30 \mathrm{~s}$ between trials, and one minute between blocks. If participants felt they needed more rest the resting period was extended. Two infra-red timing gates were placed at the starting and finishing point to measure completion time.

\subsection{Instrumentation}

Three-dimensional motion analysis was conducted by using an eight camera motion analysis system [200 Hz; Vicon Motion Analysis Systems Inc., Oxford, UK and Vicon Nexus software (version 1.8.3, Oxford, UK)]. Earlier research and review showed good measurement accuracy and test-retest reliability of the Vicon Motion Analysis System (Kadaba et al., 1989; Mcginley et al., 2009). Ground reaction force data was obtained by using two Bertec force plates (1000 Hz; Bertec Corporation, Columbus, OH). Completion

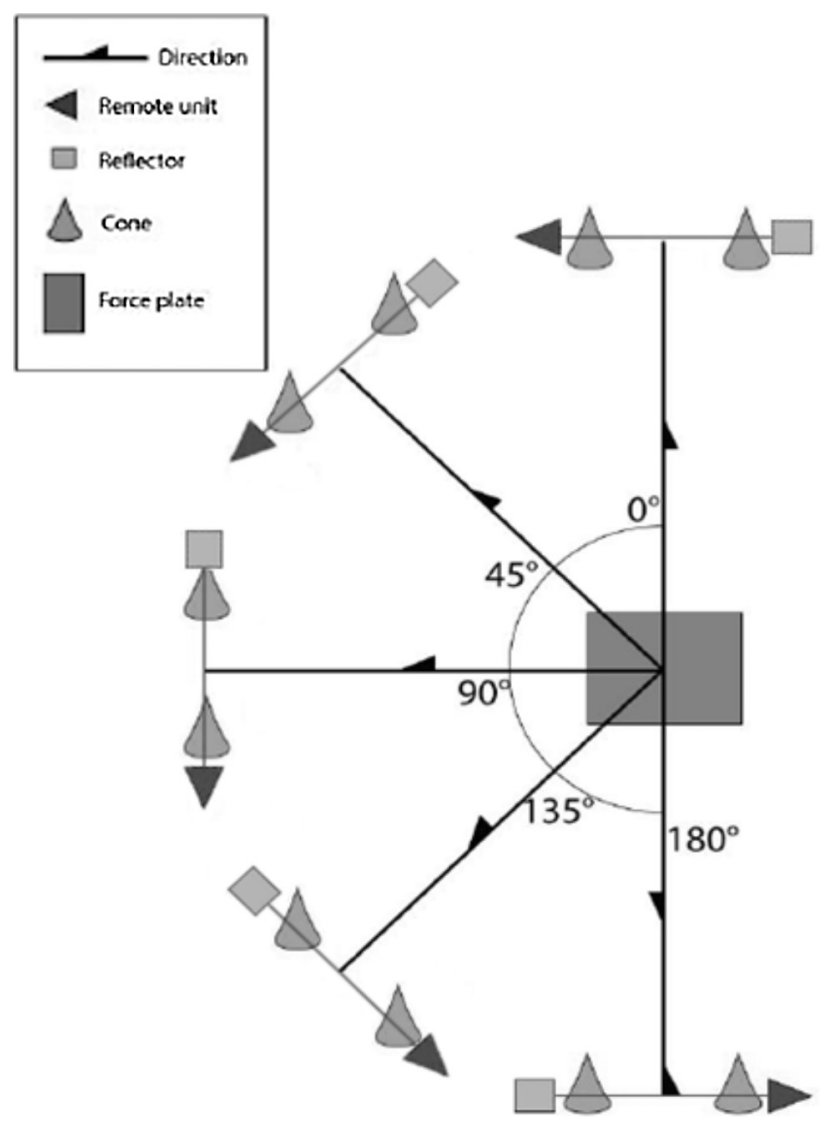

Fig. 1. Test set-up. Participants started at the bottom gate. 
Table 1

Results of repeated measures ANOVA. Means \pm SD, confidence intervals, and significance levels are displayed.

\begin{tabular}{|c|c|c|c|c|c|c|c|c|c|c|c|}
\hline & \multirow[t]{2}{*}{ Sex } & \multicolumn{2}{|l|}{$45^{\circ}$} & \multicolumn{2}{|l|}{$90^{\circ}$} & \multicolumn{2}{|l|}{$135^{\circ}$} & \multicolumn{2}{|l|}{$180^{\circ}$} & \multirow[t]{2}{*}{ p-value angle } & \multirow[t]{2}{*}{ p-value sex } \\
\hline & & Mean \pm SD & $95 \% \mathrm{CI}$ & Mean $\pm S D$ & $95 \% \mathrm{CI}$ & Mean $\pm S D$ & $95 \% \mathrm{CI}$ & Mean $\pm S D$ & $95 \% \mathrm{CI}$ & & \\
\hline \multirow[t]{2}{*}{ Knee flexion angle $\left(^{\circ}\right)$} & Male & $-43.3 \pm 7.2$ & -44.6 to -41.9 & $-42.7 \pm 11.4$ & -44.9 to -40.5 & $-43.9 \pm 7.9$ & -45.5 to -42.4 & $-42.1 \pm 8.7$ & -43.9 to -40.2 & n.s. & \multirow[t]{2}{*}{$\begin{array}{l}<0.01 \\
\mathrm{ES}=0.15\end{array}$} \\
\hline & Female & $-40.6 \pm 8.6$ & -41.9 to -39.2 & $-37.3 \pm 9.9$ & -38.9 to -35.7 & $-36.3 \pm 8.7$ & -37.7 to -34.8 & $-37.0 \pm 8.5$ & -38.3 to -35.6 & $\begin{array}{l}<0.01 \\
\mathrm{ES}=0.07\end{array}$ & \\
\hline \multirow[t]{2}{*}{ Knee flexion moment $(\mathrm{Nm} / \mathrm{kg})$} & Male & $-1.17 \pm 1.57$ & -1.47 to -0.86 & $-0.96 \pm 1.41$ & -1.23 to -0.68 & $-0.81 \pm 1.15$ & -1.03 to -0.59 & $-0.62 \pm 1.14$ & -0.85 to -0.40 & $\begin{array}{l}0.01 \\
E S=0.04\end{array}$ & \multirow[t]{2}{*}{ n.s. } \\
\hline & Female & $-1.45 \pm 1.05$ & -1.62 to -1.28 & $-0.94 \pm 1.00$ & -1.10 to -0.78 & $-0.57 \pm 0.78$ & -0.69 to -0.44 & $-0.63 \pm 0.73$ & -0.75 to -0.52 & $\begin{array}{l}<0.01 \\
\mathrm{ES}=0.19\end{array}$ & \\
\hline \multirow[t]{2}{*}{ Knee varus/valgus moment $(\mathrm{Nm} / \mathrm{kg})$} & Male & $0.13 \pm 0.85$ & $-0.03-0.29$ & $-0.84 \pm 0.82$ & -1.00 to -0.68 & $-0.91 \pm 0.60$ & -1.03 to -0.80 & $-0.96 \pm 0.64$ & -1.09 to -0.84 & $\begin{array}{l}<0.01 \\
\mathrm{ES}=0.37\end{array}$ & \multirow[t]{2}{*}{$\begin{array}{l}<0.01 \\
\mathrm{ES}=0.06\end{array}$} \\
\hline & Female & $0.07 \pm 0.50$ & $-0.01-0.15$ & $-0.57 \pm 0.48$ & -0.65 to -0.50 & $-0.71 \pm 0.56$ & $-0.80-0.62$ & $-0.60 \pm 0.56$ & $-0.70-0.51$ & $\begin{array}{l}<0.01 \\
\mathrm{ES}=0.36\end{array}$ & \\
\hline \multirow[t]{2}{*}{ Knee flexion RoM $\left(^{\circ}\right)$} & Male & $-11.3 \pm 7.6$ & -12.9 to -9.8 & $-17.5 \pm 12.2$ & -20.0 to -15.0 & $-14.7 \pm 9.0$ & -16.5 to -12.8 & $-12.7 \pm 8.4$ & -14.4 to -11.0 & $\begin{array}{l}<0.01 \\
\mathrm{ES}=0.09\end{array}$ & \multirow[t]{2}{*}{ n.s. } \\
\hline & Female & $-12.5 \pm 9.0$ & -14.0 to -10.9 & $-14.7 \pm 8.7$ & -16.2 to -13.1 & $-13.6 \pm 7.1$ & -14.9 to -12.4 & $-14.6 \pm 7.7$ & -15.9 to -13.3 & $\begin{array}{l}0.03 \\
\mathrm{ES}=0.02\end{array}$ & \\
\hline \multirow[t]{2}{*}{ vGRF (N/kg) } & Male & $22.7 \pm 6.3$ & $21.5-23.9$ & $19.1 \pm 5.0$ & $18.2-20.1$ & $15.9 \pm 3.9$ & $15.2-16.7$ & $16.2 \pm 4.1$ & $15.4-17.0$ & $\begin{array}{l}<0.01 \\
\mathrm{ES}=0.32\end{array}$ & \multirow[t]{2}{*}{$\begin{array}{l}<0.01 \\
E S=0.11\end{array}$} \\
\hline & Female & $18.5 \pm 4.6$ & $17.7-19.2$ & $15.9 \pm 4.2$ & $15.2-16.6$ & $16.1 \pm 4.1$ & $15.4-16.8$ & $15.6 \pm 4.5$ & $14.9-16.3$ & $\begin{array}{l}<0.01 \\
\mathrm{ES}=0.11\end{array}$ & \\
\hline \multirow[t]{2}{*}{ Completion time (s) } & Male & $2.15 \pm 0.11$ & $2.13-2.17$ & $2.60 \pm 0.16$ & $2.57-2.63$ & $2.83 \pm 0.18$ & $2.73-2.86$ & $2.98 \pm 0.19$ & $2.94-3.02$ & $\begin{array}{l}<0.01 \\
\mathrm{ES}=0.87\end{array}$ & \multirow[t]{2}{*}{$\begin{array}{l}<0.01 \\
E S=0.42\end{array}$} \\
\hline & Female & $2.36 \pm 0.10$ & $2.35-2.38$ & $2.75 \pm 0.12$ & $2.73-2.77$ & $3.00 \pm 0.14$ & $2.98-3.03$ & $3.15 \pm 0.15$ & $3.13-3.17$ & $\begin{array}{l}<0.01 \\
\mathrm{ES}=0.94\end{array}$ & \\
\hline
\end{tabular}

$\mathrm{s}=$ seconds,${ }^{\circ}=$ degrees, $\mathrm{Nm} / \mathrm{kg}=$ Newtonmeter $/ \mathrm{kilogram}, \mathrm{RoM}=$ range of motion, $\mathrm{vGRF}=$ vertical ground reaction force, $\mathrm{N} / \mathrm{kg}=\mathrm{Newton} / \mathrm{kilogram}$. 

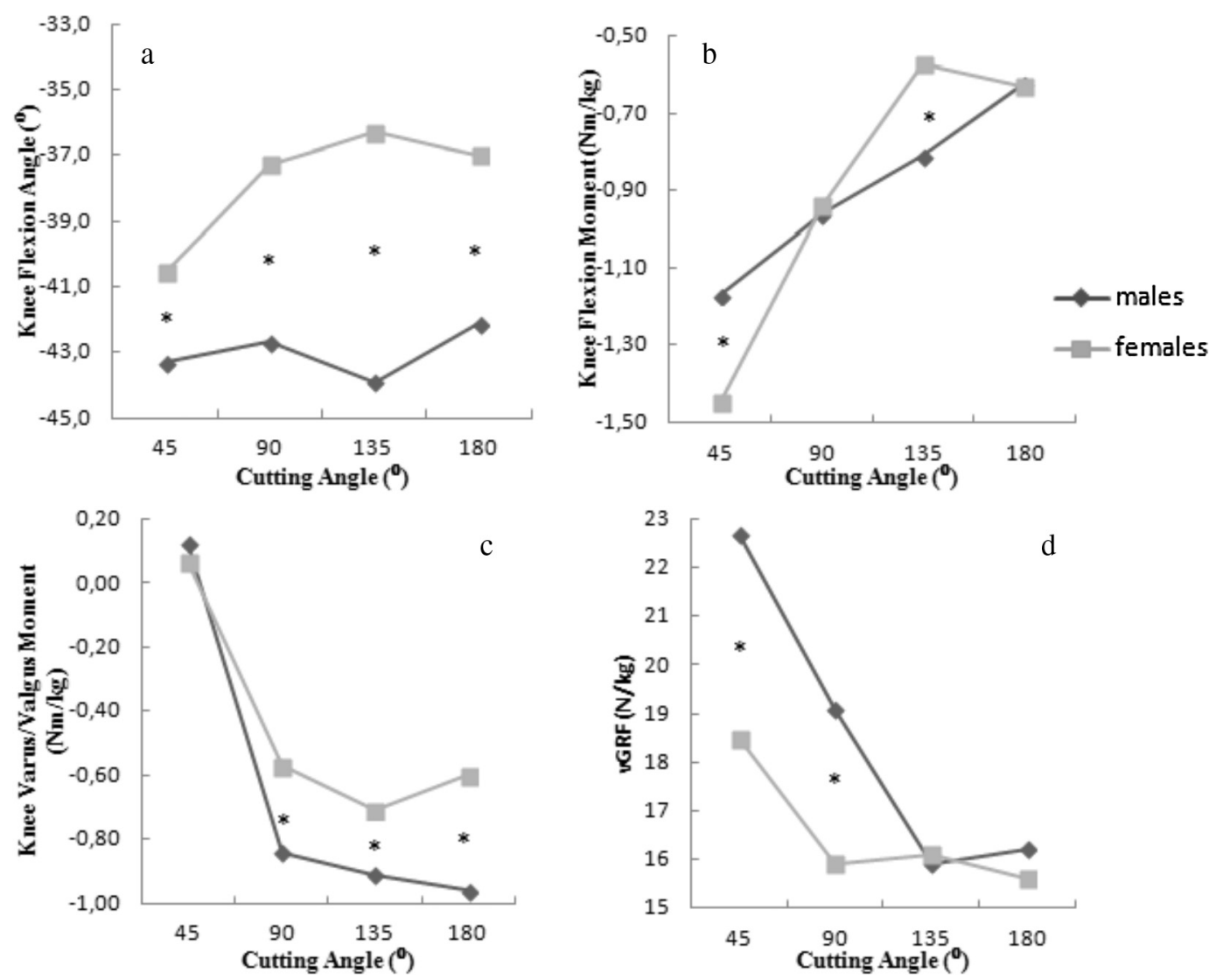

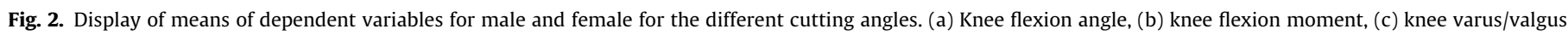
moment, (d) vGRF. Significant sex differences are marked by with an asterisk.

time was acquired using two Smartspeed Pro time gates (Fusion Sport, Australia).

\subsection{Data analysis}

Primary outcome variables for this study were vGRF, knee flexion angle, knee flexion ROM, knee flexion moment, knee varus/valgus moment and completion time. All the kinetic and kinematic values were expressed at peak varus/valgus moment during the deceleration phase, because this parameter has been associated with an increased ACL injury risk (Leppanen et al., 2017; Boden et al., 2000). ROM was determined by the value at peak varus/valgus minus the value at initial contact. Moments were normalized to body weight. All joint angles, moments and ROM's were calculated using Matlab 7.11 (The MathWorks Inc., Natick, MA). Only the kinematics and kinetics of the right leg were calculated. Force plate and kinetic data were filtered using a fourth order low pass Butterworth filter with a cut off frequency of $10 \mathrm{~Hz}$.

Only the trials where the participant made a cut where analysed in this study, so the trials with a cut of $45^{\circ}, 90^{\circ}, 135^{\circ}$ and $180^{\circ}$. To assess the effect of cutting angle and sex on kinematics and kinetics a $4 \times 2$ repeated measures ANOVA was used. GreenhouseGeisser adjustments were used if the sphericity was violated. The repeated measures ANOVA was followed by a post hoc comparisons (Bonferoni) comparisons with an alpha-level of $\alpha \leq 0.05 \mathrm{a}$ priori. Results are reported with one decimal (Windolf et al., 2008). Based on number of participants and pooled standard deviation, effect sizes (ES) were calculated for all comparisons. Cohen's d (small $(d \leq 0.2)$, moderate $(0.2 \geq d \leq 0.8)$ and large $(\mathrm{d} \geq 0.8)$ ) was calculated to determine the ES of the found significant effects (Cohen, 2013). This paper was written according to the STROBE checklist.

\section{Results}

The kinematics and kinetics for each cutting angle are presented in Table 1 and in Fig. 2. Significant post hoc tests are displayed in Table 2.

The results showed that males performed all cutting angles with the same knee flexion angle. Females, however, showed lower knee flexion angles when cutting to $90^{\circ}, 135^{\circ}$ and $180^{\circ}$ cutting angles compared to the $45^{\circ}$ cutting angle ( $p<0.01$, ES $=0.07$ ). Further, the results showed that at all cutting angles, males showed greater knee flexion angles than females $(\mathrm{p}<0.01$, ES $=0.15)$.

For males the knee flexion moment approached $0 \mathrm{Nm} / \mathrm{kg}$ as the cutting angle increases $\left(45^{\circ}\right.$ versus $180^{\circ}(\mathrm{p}=0.01$, ES $=0.04)$ ). Also in females the knee flexion moment showed a decrease in knee flexion moment when cutting to respectively $45^{\circ}, 90^{\circ}$ and $135^{\circ}$ ( $p<0.01$, ES $=0.19$ ). No significant difference was found for knee flexion moment between the $135^{\circ}$ and $180^{\circ}$. Knee flexion moment did not significantly differ between males and females.

Both males and females showed significant differences in knee valgus moment $(p<0.01$, ES $=0.37$ and $p<0.01, E S=0.36)$. Both sexes had greater knee valgus moments in the cutting angles of $90^{\circ}, 135^{\circ}$ and $180^{\circ}$ compared to the $45^{\circ}$ cutting angle (all $\mathrm{p}<0.01$ ). Within sex, the knee varus/valgus moment did not differ across the angles of $90^{\circ}, 135^{\circ}$ and $180^{\circ}$. The knee varus/valgus moment stabilized when the participants made cuts with angles of $90^{\circ}$ or higher (Fig. 2c). Regarding sex, males had greater knee valgus moments than females at $90^{\circ}, 135^{\circ}$ and $180^{\circ}(\mathrm{p}<0.01, \mathrm{ES}=0.06)$.

The knee flexion RoM for males differed across cutting angles ( $p<0.01$, ES $=0.09$ ), but no clear trend was visible in the data. In females a significant effect in knee flexion RoM was found $(p=0.03, E S=0.02)$. However, no differences across cutting angles in the post hoc test were found with regard to knee flexion RoM. 
Table 2

Significant post hoc tests repeated measures ANOVA.

\begin{tabular}{|c|c|c|}
\hline & Sex & Significant post hoc tests \\
\hline Completion time (s) & Female & $\begin{array}{l}45 \mathrm{vs} 90 \mathrm{p}<0.001 \\
45 \mathrm{vs} 135 \mathrm{p}<0.01 \\
45 \mathrm{vs} 180 \mathrm{p}<0.01 \\
90 \mathrm{vs} 135 \mathrm{p}<0.01 \\
90 \mathrm{vs} 180 \mathrm{p}<0.01 \\
135 \mathrm{vs} 180 \mathrm{p}<0.01 \\
45 \mathrm{vs} 90 \mathrm{p}<0.001 \\
45 \mathrm{vs} 135 \mathrm{p}<0.01 \\
45 \mathrm{vs} 180 \mathrm{p}<0.01 \\
90 \mathrm{vs} 135 \mathrm{p}<0.01 \\
90 \mathrm{vs} 180 \mathrm{p}<0.01 \\
135 \mathrm{vs} 180 \mathrm{p}<0.01\end{array}$ \\
\hline Knee flexion angle $\left(^{\circ}\right)$ & $\begin{array}{l}\text { Male } \\
\text { Female }\end{array}$ & $\begin{array}{l}45 v s 90 \mathrm{p}<0.01 \\
45 \mathrm{vs} 135 \mathrm{p}<0.01 \\
45 \mathrm{vs} 180 \mathrm{p}<0.01\end{array}$ \\
\hline Knee flexion moment $(\mathrm{Nm} / \mathrm{kg})$ & $\begin{array}{l}\text { Male } \\
\text { Female }\end{array}$ & $\begin{array}{l}45 \mathrm{vs} 180 \mathrm{p}=0.01 \\
45 \mathrm{vs} 90 \mathrm{p}<0.01 \\
45 \mathrm{vs} 135 \mathrm{p}<0.01 \\
45 \mathrm{vs} 180 \mathrm{p}<0.01 \\
90 \mathrm{vs} 135 \mathrm{p}<0.01 \\
90 \mathrm{vs} 180 \mathrm{p}<0.01\end{array}$ \\
\hline Knee varus/valgus moment $(\mathrm{Nm} / \mathrm{kg})$ & $\begin{array}{l}\text { Male } \\
\text { Female }\end{array}$ & $\begin{array}{l}45 \mathrm{vs} 90 \mathrm{p}<0.01 \\
45 \mathrm{vs} 135 \mathrm{p}<0.01 \\
45 \mathrm{vs} 180 \mathrm{p}<0.01 \\
45 \mathrm{vs} 90 \mathrm{p}<0.01 \\
45 \mathrm{vs} 135 \mathrm{p}<0.01 \\
45 \mathrm{vs} 180 \mathrm{p}<0.01\end{array}$ \\
\hline Knee flexion RoM $\left(^{\circ}\right)$ & $\begin{array}{l}\text { Male } \\
\text { Female }\end{array}$ & $\begin{array}{l}45 v s 90 \mathrm{p}<0.01 \\
45 \mathrm{vs} 135 \mathrm{p}<0.01 \\
90 \mathrm{vs} 180 \mathrm{p}<0.01 \\
\text { n.s. }\end{array}$ \\
\hline vGRF (N/kg) & Female & $\begin{array}{l}45 \mathrm{vs} 90 \mathrm{p}<0.01 \\
45 \mathrm{vs} 135 \mathrm{p}<0.01 \\
45 \mathrm{vs} 180 \mathrm{p}<0.01 \\
90 \mathrm{vs} 135 \mathrm{p}<0.01 \\
90 \mathrm{vs} 180 \mathrm{p}<0.01 \\
45 \mathrm{vs} 90 \mathrm{p}<0.001 \\
45 \mathrm{vs} 135 \mathrm{p}<0.01 \\
45 \mathrm{v} 180 \mathrm{p}<0.01\end{array}$ \\
\hline
\end{tabular}

$\mathrm{s}=$ seconds, ${ }^{\circ}=$ degrees, $\mathrm{Nm} / \mathrm{kg}=$ Newtonmeter $/$ kilogram, RoM = range of motion, $\mathrm{vGRF}=$ vertical ground reaction force, $\mathrm{N} / \mathrm{kg}=$ Newton/kilogram.

No differences in knee flexion RoM were found between males and females.

Males and females showed significant lower vGRF in the sharper angles (both $\mathrm{p}<0.01, \mathrm{ES}=0.32$ and $\mathrm{ES}=0.11$ ). In males these differences were significantly smaller when comparing $135^{\circ}$ and $180^{\circ}$ to $45^{\circ}$ and $90^{\circ}$, where in females also the $90^{\circ}$ was significantly smaller compared the $45^{\circ}$. In general males had greater vGRF than females $(\mathrm{p}<0.01, \mathrm{ES}=0.11)$.

The results further show that the completion times within males are significantly faster than within females $(p<0.01$, $\mathrm{ES}=0.42$ ). For both sexes the completion time got higher when the cutting angle increased (both $\mathrm{p}<0.01, \mathrm{ES}=0.87$ and $\mathrm{ES}=0.94$ ). The average speed for males was $4.7 \mathrm{~m} / \mathrm{s}, 3.8 \mathrm{~m} / \mathrm{s}$, $3.5 \mathrm{~m} / \mathrm{s}$ and $3.4 \mathrm{~m} / \mathrm{s}$, respectively. For females these averages were $4.2 \mathrm{~m} / \mathrm{s}, 3.6 \mathrm{~m} / \mathrm{s}, 3.3 \mathrm{~m} / \mathrm{s}$ and $3.2 \mathrm{~m} / \mathrm{s}$.

\section{Discussion}

The main aim of this study was to investigate the effects of cutting angle on knee kinetics and kinematics.

In terms of knee flexion angle results show that males used a larger knee flexion than females regardless of cutting angle.
Females used a smaller knee flexion when comparing $90^{\circ}, 135^{\circ}$ and $180^{\circ}$ to the $45^{\circ}$ condition. With smaller knee angles, the quadriceps place more strain on the ACL (Nisell, 1985). Cortes and colleagues found a similar pattern (Cortes et al., 2011). They found average knee flexion angles of $-53.9^{\circ}$ and $-41.2^{\circ}$ when cutting towards $45^{\circ}$ and $180^{\circ}$ respectively, similar to our study. They, however, found this difference at peak vGRF whereas in the current study the difference was found at peak knee varus/valgus moment. This could also explain why they found larger knee flexion angles in general. The decrease in knee flexion angle when cutting to sharper angles could be a result of a smaller knee flexion moment when cutting to sharper angles. Also a lower approach speed in the terminal steps during the sharper angles could result in a lower knee flexion moment. The smaller knee flexion angles in females can be explained by the fact that females have proportionally weaker quadriceps musculature. A cut with a greater knee angle demands a lot of strength from the quadriceps muscles, which is better handled by males compared to females, even along with lower completion time. Another explanation could be that female use a more ligament dominant landing, resulting in lower knee flexion angles (Myer et al., 2011).

In contrary to the knee flexion moment, the knee varus/valgus moment did increase when cutting to sharper angles. In the condition of $45^{\circ}$ there is still a slight varus moment, but when cutting to $90^{\circ}$ or higher, athletes depict a valgus moment. This means that in these conditions there could be a larger strain on the ACL in terms of knee varus/valgus moment (Ford et al., 2003). Remarkably the biggest change in varus/valgus moment was between cutting angles of $45^{\circ}$ and $90^{\circ}$. For both sexes, when cutting to sharper angles than $90^{\circ}$ the valgus moment stabilizes around a certain value. It could be that athletes subconsciously restrain this moment from becoming greater when cutting to sharper angles. From the results it seems that females stabilize the knee valgus moment at a smaller value compared to males. Future research should focus on how athletes stabilize this moment when cutting to sharper angles, by for example also taking into account trunk, hip and ankle kinetics and kinematics. This knowledge could be of great value to ACL injury prevention programs. Jamison and colleagues already found that knee load is dependent on trunk control (Jamison et al., 2013). Also, preliminary observation of our trunk data show that males use greater trunk flexion angles when cutting towards sharper angles. Another possible explanation of the stabilization after can be found in earlier research (Dempsey et al., 2012). Their study found that knee valgus moments were related to the orientation of the knee relative to the direction of travel. It is likely that from cuts of $90^{\circ}$ and greater the knee is orientated close to perpendicular to the direction of initial where it has the greatest potential to experience a valgus moment. The findings of knee varus/valgus moment are related with the findings of vGRF. The frontal plane moment increased with sharper cuts, where participants decreased their vGRF. In other words, when the athletes leans more into the corner to accomplish the greater redirection demands, their perpendicular distance to the GRF is larger, increasing their angular acceleration. Further, it was found that athletes had smaller vGRF in sharper angles. This can be explained by the fact that when cutting to larger angles the athletes have to lean more towards the corner. The vGRF becomes less perpendicular in respect to the ground, as the center of pressure is further away from the center of mass.

The knee flexion RoM showed some differences across the cutting angles, but no clear relation was found. No significant differences between sexes were found. It is remarkable that considerably smaller knee flexion RoM was found than in other studies (Havens and Sigward, 2015a, b; Cortes et al., 2011; Benjaminse et al., 2015b). This could be because in the current study participants completed the task at maximum effort whereas 
in the other studies the participants used a predetermined approach and exit speed. This would imply that cutting at maximum effort results in suboptimal load dissemination (Dai et al., 2014). Another explanation might be that in the current study knee flexion RoM was calculated from the initial contact till the peak knee varus/valgus moment. This could result in less time for the athlete to flex the knee.

This was the first study directly comparing how athletes of both sexes adjust whole body mechanics when changing direction in a range of various cutting angles, included both outcome measure on movement technique, with a pressure on performance. Our results are comparable to those of earlier research where no linear increase of demands is seen when the cutting angle increases (Havens and Sigward, 2015a, b). Cuts from $90^{\circ}$ and larger demand more braking and translation skills than the $45^{\circ}$ cut. Generally speaking, sagittal plane demands decrease and frontal plane demands increase with sharper changes of direction. We did see though that males were able to keep their knee flexion large at all cutting angles.

Results should be interpreted with caution, since testing took place in a controlled environment. The results will probably be different when performing the cuts during a on-field training session or match. Also in this study only the dominant leg was taken into account, but earlier research showed no difference in kinetics and kinematics between the dominant and non-dominant leg in cutting tasks (Bencke et al., 2013; Matava et al., 2002). This study included recreational athletes and results cannot be transferred to elite athletes (Abernethy et al., 1999). Further in this study a preplanned task was used, it is expected that joint loads will increase during reactive tasks (Besier et al., 2001). We therefore only assessed the participant's physical abilities to change direction and not respond to a sport-specific stimulus. For future research it is encouraged to include whole body or joint mechanics associated with the entire stance phase, and even the approach steps, will provide additional insight into technique used for the various directional changes. Even though power was sufficient in this study, findings need to be interpreted with respect to the effect sizes.

The current study helped to gain more insight in differences between sex and cutting angles on knee kinetics and kinematics during side step cutting. The findings have implications for ACL injury prevention programs, where it is important to maintain performance, i.e. running speed, while improving technique (Benjaminse et al., 2015b; Vescovi and VanHeest, 2010). For example, start at sub maximal speed when practicing sharp cuts, considering the increase in knee valgus moment during cuts at $>45^{\circ}$. Also, since the results showed differences between males and females, this could also be taken into account by for example using different instructions or feedback. For example, to improve whole body movement technique, a sex specific video of an expert athlete performing the cut optimally can be shown to the athlete while instructing to mimic the movement to the best of their ability (Benjaminse et al., 2015a). Or help the athlete improving cutting technique by stimulating the athlete to move the trunk lean and rotation to the direction of movement, for example by moving towards an external goal (Jamison et al., 2013; Dempsey et al., 2007). To accomplish the video feedback, verbal instructions to improve technique and reduce knee load, could be: 'Move your body towards goal when making the cut' or 'Push yourself as hard as possible off the ground when making the cut'. Lastly, it should also be advised to include 'real-life' factors when practicing tasks, such as reacting to a stimulus and changing direction while dribbling a ball. This increases validity and transfer of learned skills to the field, as this also involves processing of visual information, anticipation, response selection and motor programming (Sheppard and Young, 2006).

\section{Conclusion}

It can be concluded that different cutting angles demand different knee kinematics and kinetics. Sharper cutting angles place the knee more at risk. However, females and males handle this differently, which has implications for injury prevention. It can be concluded that cutting to different angles has distinct kinetic and kinematic characteristics. In general males used greater knee flexion angles than females. In males no effect of cutting angle on knee flexion angle was found. Males had higher knee valgus moments during the cuts of $90^{\circ}, 135^{\circ}$ and $180^{\circ}$. For both sexes knee flexion moment got smaller when cutting to sharper angles. But no effect of sex on the knee flexion moment was found.

In terms of knee varus/valgus, an important ACL injury predictor, athletes experience greater knee varus/valgus moments in sharper cutting angles compared to $45^{\circ}$ cutting angle, and therefore are at greater risk at these angles. This is in line with what was hypothesized. But it seems athletes limit these moments to a certain value and no increased risk was found when cutting to angles beyond $90^{\circ}$.

\section{Acknowledgements}

We thank Prof. dr. Bert Otten for his assistance with Matlab.

\section{Conflict of interest}

All procedures performed in studies involving human participants were in accordance with the ethical standards of the institutional and/or national research committee and with the 1964 Helsinki declaration and its later amendments or comparable ethical standards. The authors have no conflicts of interest that are directly relevant to the content of this article.

\section{References}

Abernethy, B., Wood, J.M., Parks, S., 1999. Can the anticipatory skills of experts be learned by novices? Res. Q. Exerc. Sport 70, 313-318.

Bencke, J., Curtis, D., Krogshede, C., Jensen, L.K., Bandholm, T., Zebis, M.K., 2013 Biomechanical evaluation of the side-cutting manoeuvre associated with ACL injury in young female handball players. Knee Surg. Sports Traumatol. Arthrosc. 21, 1876-1881.

Benjaminse, A., Gokeler, A., Dowling, A.V., Faigenbaum, A., Ford, K.R., Hewett, T.E., Onate, J.A., Otten, B., Myer, G.D., 2015a. Optimization of the anterior cruciate ligament injury prevention paradigm: novel feedback techniques to enhance motor learning and reduce injury risk. J. Orthop. Sport. Phys. 45, 170-182.

Benjaminse, A., Otten, B., Gokeler, A., Diercks, R.L., Lemmink, K.A., 2015b. Motor learning strategies in basketball players and its implications for ACL injury prevention: a randomized controlled trial. Knee Surg. Sports Traumatol Arthrosc. 25, 2365-2376.

Besier, T.F., Lloyd, D.G., Ackland, T.R., Cochrane, J.L., 2001. Anticipatory effects on knee joint loading during running and cutting maneuvers. Med. Sci. Sports Exerc. 33, 1176-1181.

Bloomfield, J., Polman, R., O'Donoghue, P., 2007a. Turning movements performed during FA Premier League soccer matches. J. Sports Sci. Med. 6, 9-10.

Bloomfield, J., Polman, R., O’Donoghue, P., 2007b. Physical demands of different positions in FA premier league soccer. J. Sports Sci. Med. 6, 63-70.

Boden, B.P., Dean, G.S., Feagin, J.A., Garrett, W.E., 2000. Mechanisms of anterior cruciate ligament injury. Orthopedics 23, 573-578.

Cochrane, J.L., Lloyd, D.G., Buttfield, A., Seward, H., McGivern, J., 2007. Characteristics of anterior cruciate ligament injuries in Australian football. J. Sci. Med. Sport 10, 96-104.

Cohen, J., 2013. Statistical power analysis for the behavioral sciences. Erlbaum (Eds.), Hillsdale, NJ.

Cortes, N., Onate, J., Van Lunen, B., 2011. Pivot task increases knee frontal plane loading compared with sidestep and drop-jump. J. Sports Sci. 29, 83-92.

Dai, B., Butler, R., Garrett, W., Queen, R., 2014. Using ground reaction force to predict knee kinetic asymmetry following anterior cruciate ligament reconstruction. Scand. J. Med. Sci. Sports 24, 974-981.

Dempsey, A.R., Lloyd, D.G., Elliott, B.C., Steele, J.R., Munro, B.J., Russo, K.A., 2007. The effect of technique change on knee loads during sidestep cutting. Med. Sci. Sports Exerc. 39, 1765-1773. 
Dempsey, A.R., Elliott, B.C., Munro, B.J., Steele, J.R., Lloyd, D.G., 2012. Whole body kinematics and knee moments that occur during an overhead catch and landing task in sport. Clin. Biomech. 27, 466-474.

Ford, K.R., Myer, G.D., Hewett, T.E., 2003. Valgus knee motion during landing in high school female and male basketball players. Med. Sci. Sports Exerc. 35, 17451750.

Greig, M., 2009. The influence of soccer-specific activity on the kinematics of an agility sprint. Eur. J. Sport Sci. 9, 23-33.

Hase, K., Stein, R.B., 1999. Turning strategies during human walking. J. Neurophysiol. 81, 2914-2922.

Havens, K.L., Sigward, S.M., 2015a. Joint and segmental mechanics differ between cutting maneuvers in skilled athletes. Gait Posture 41, 33-38.

Havens, K.L., Sigward, S.M., 2015b. Cutting mechanics: relation to performance and anterior cruciate ligament injury risk. Med. Sci. Sports Exerc. 47, 818-824.

Jamison, S.T., McNally, M.P., Schmitt, L.C., Chaudhari, A.M., 2013. The effects of core muscle activation on dynamic trunk position and knee abduction moments: implications for ACL injury. J. Biomech. 46, 2236-2241.

Kadaba, M., Ramakrishnan, H., Wootten, M., Gainey, J., Gorton, G., Cochran, G., 1989. Repeatability of kinematic, kinetic, and electromyographic data in normal adult gait. J. Orthop. Res. 7, 849-860.

Leppanen, M., Pasanen, K., Kujala, U.M., Vasankari, T., Kannus, P., Ayramo, S., Krosshaug, T., Bahr, R., Avela, J., Perttunen, J., Parkkari, J., 2017. Stiff landings are associated with increased ACL injury risk in young female basketball and floorball players. Am. J. Sports Med. 45, 386-393.

Matava, M.J., Freehill, A.K., Grutzner, S., Shannon, W., 2002. Limb dominance as a potential etiologic factor in noncontact anterior cruciate ligament tears. J. Knee Surg. 15, 11-16.

McGinley, J.L., Baker, R., Wolfe, R., Morris, M.E., 2009. The reliability of threedimensional kinematic gait measurements: a systematic review. Gait Posture 29, 360-369.

McLean, S.G., Lipfert, S.W., Van Den Bogert, A.J., 2004. Effect of gender and defensive opponent on the biomechanics of sidestep cutting. Med. Sci. Sports Exerc. 36 1008.
Myer, G.D., Brent, J.L., Ford, K.R., Hewett, T.E., 2011. Real-time assessment and neuromuscular training feedback techniques to prevent ACL injury in female athletes. Strength Cond. J. 33, 21-35.

Newell, K.M., 1996. Change in movement and skill: Learning, retention, and transfer. In: Latash, M.L., Turvey, M.T. (Eds.), Dexterity and its development. Erlbaum, Mahwah, NJ, pp. 393-430.

Newell, K.M., Slifkin, A.B., 1998. The nature of movement variability. In: Piek, J.P. (Ed.), Motor behavior and human skill: a multidisciplinary perspective. Human Kinetics, Champaign, IL, pp. 143-160.

Nisell, R., 1985. Mechanics of the knee: a study of joint and muscle load with clinical applications. Acta Orthop. Scand. 56, 1-42.

Olsen, O.E., Myklebust, G., Engebretsen, L., Bahr, R., 2004. Injury mechanisms for anterior cruciate ligament injuries in team handball: a systematic video analysis. Am. J. Sports Med. 32, 1002-1012.

Reilly, T., Williams, A.M., Nevill, A., Franks, A., 2000. A multidisciplinary approach to talent identification in soccer. J. Sports Sci. 18, 695-702.

Sekulic, D., Pehar, M., Krolo, A., Spasic, M., Uljevic, O., Calleja-Gonzalez, J., Sattler, T., 2016. Evaluation of basketball-specific agility; applicability of pre-planned and non-planned agility performances for differentiating playing positions and playing levels. J. Strength Cond. Res. 31, 2278-2288.

Sheppard, J.M., Young, W.B., 2006. Agility literature review: classifications, training and testing. J. Sports Sci. 24, 919-932.

Sigward, S.M., Cesar, G.M., Havens, K.L., 2015. Predictors of frontal plane knee moments during side-step cutting to 45 and 110 degrees in men and women: implications for anterior cruciate ligament injury. Clin. J. Sport Med. 25, 529534.

Vescovi, J.D., VanHeest, J.L., 2010. Effects of an anterior cruciate ligament injury prevention program on performance in adolescent female soccer players. Scand. J. Med. Sci. Sports 20, 394-402.

Windolf, M., Götzen, N., Morlock, M., 2008. Systematic accuracy and precision analysis of video motion capturing systems-exemplified on the Vicon-460 system. J. Biomech. 41, 2776-2780. 